\title{
欧美军用红外通用组件技术的发展现状及趋势
}

\author{
张坤杰 \\ (昆明物理研究所, 云南 昆明 650223)
}

\begin{abstract}
摘要: 在军用红外技术的早期发展阶段, 欧美基于热成像系统关键部件的通用化形成了通用组件概念。 通用组件虽然减少了工程上选择不同技术方案的余地, 但是降低了热成像系统的研发成本和使用维护 费用, 有利于批量生产。美国以一代通用组件为基础形成了 SADA 系列组件, 在 SADA 基础上推出 的水平技术集成（Horizontal Technology Integration，HTI）项目，实现了军用热像仪的批量生产和装 备。介绍了欧美通用组件技术的发展历史和现状。随着探测器、微电子等相关技术的发展, 通用组件 已经从原来的 Common Module 转变为 Module。
\end{abstract}

关键词: 通用组件; 热像仪; 军用红外技术

中图分类号：TN216 文献标识码：A 文章编号：1001-8891(2020)07-0697-05

\section{Military Status and Trends of Infrared Common Modules in Europe and America}

ZHANG Kunjie

(Kunming Institute of Physics, Kunming 650223, China)

\begin{abstract}
In the early stages of military infrared technology development, common modules were formed by standardizing the key components of thermal imaging systems in Europe and America. These common modules decreased the scope of choice between different technical proposals. However, it reduced the costs of researching, developing, operating, and maintaining infrared systems, and was beneficial to mass production. The SADA-series assembly was established in America based on the first-generation common module. The implementation of the horizontal technology integration (HTI) program based on the SADA assembly met the requirements of mass production and equipment for military thermal imaging. In this paper, the history and status of the common module in Europe and America is introduced. Common modules have become critical to the advancement of detector, microelectronics, and other related technologies.
\end{abstract}

Key words: common module, thermal imager, military infrared technology,

\section{0 引言}

红外技术是典型的军民两用技术。二战后, 军用 红外技术在以美国为代表的西方发达国家得到快速 发展。以美国为例, 先后投入巨资实施了 70 多项军 用红外项目，装备范围包括坦克、车辆、飞机、舰船、 轻武器瞄准镜、战术导弹、战略导弹、反导系统、火 控系统、战场监视和观察设备, 以及单兵侦察设备等。 在军用红外技术发展的早期阶段, 因为军方对每一种 热成像系统的采购都是独立进行的, 各系统的主要部 分都存在着重复设计、性能大同小异等情况, 导致采 购数量停留在较低水平, 成本和可靠性方面都无法得 到批量生产的优势, 为了解决这一问题, 产生了通用
组件概念。经过几十年的发展，欧美国家已经形成了 高水平的通用组件技术（群）。本文介绍了欧美通用 组件技术的发展及趋势。

\section{1 一代通用组件的产生}

1972 年, 美国德州仪器（Texas Instruments，TI） 公司的研发人员发现, 在热成像系统的大量应用中, 前端光学系统和末端显示器是需要定制的必要部件, 约占系统成本的 $35 \%$, 所有热成像系统都需要使用的 探测器、扫描器和信号处理电路等关键部件不需要定 制, 成本约占 $45 \%$ ～70\%, 将关键部件标准化, 在不 同的装备平台之间形成通用组件，可降低制造成本。 通用组件 (Common Module) 是标准化的光学和电子 
学套件, 将其作为每一特殊热成像系统的核心部件, 再根据装备平台的指定要求, 配备所需的前端光学系 统和显示器, 组成构形、视场、显示与性能各不相同 的完整热像仪, 其核心思想就是 “关键部件通用化” ${ }^{[1]}$ 。 由于组件标准化, 可以减少维护、补给、训练等其它 费用支出, 满足系统性能、封装限制和维护要求, 具 有较高的有效性、可靠性和可维护性 ${ }^{[2]}$ 。

根据美国军方需求, 美国第一代通用组件诞生于 1976 年, 共分为探测器/杜瓦瓶、制冷机、扫描器、 热成像仪、发光二级管阵列、可见光准直器、前置放 大器、偏置调节器、主放大器/驱动器、扫描/隔行电 子学和辅助控制电子学 11 个部分 ${ }^{[1]}$ 。采用并扫模式, 低、中、高三级性能, 分别使用 60 元、 120 元和 180 元碲镉录 (Mercury Cadmium Telluride, MCT) 探测 器。60 元探测器一般用于反坦克导弹火控瞄准镜, 120 元探测器一般用于坦克瞄准镜系统, 180 元探测器一 般用于机载热成像系统。

与此同时, 英国于 1976 年批准了通用组件方案, 1982 年投产。英国热成像通用组件最初分为 3 类, I 类用于便携式热成像系统, 采用双排 23 元 MCT 探测 器; II 类用于与电视兼容的监视系统; III 类用于较敏 感的飞行员辅助观察系统。20 世纪 80 年代, 英国研 究人员研发出基于 $8 \sim 13 \mu \mathrm{m}$ SPRITE (Signal Processing In The Element) 探测器的非直视型热像仪, 可在探测器内完成信号处理, 不需要时间延迟积分电 路。I 类便携式直视型热成像通用组件不能利用 SPRITE 探测器的成像优势。带有大口径光学系统的 SPRITE 热像仪具有较好灵敏度, 所以更高灵敏度的 III 类扫描器组件只做了样机, 没有投入生产。因此, II 类通用组件成为基于 SPRITE 探测器的非直视型热成 像通用组件 (Thermal Imaging Common Modules, TICM II）, 由远焦系统、宽角度、双扫描机构模块、1 个探 测器镜头和 8 条 MCT SPRITE 探测器组成, 8 条 SPRITE 的性能相当于 100 元以上的多元探测器 ${ }^{[3-4]}$ 。

法国于 1975 年开始对通用组件进行预研, 1980 1981 年完成样机研制和通用组件系统评价, 1985 年 中期投产，国家批准建立生产线，批量生产通用组件、 组装应用系统, 其应用平台和领域则根据法国三军的 各种合同和要求进行。1987 年交付第一批通用组件产 品。采用串并扫模式, 使用 $5 \times 11$ 元 MCT 光伏型探 测器, 包括探测器、制冷机、扫描器、LED 显示器、 LED 电子单元、小型 CRT 监视器、线性电子单元、 信号处理、传感头自动测试和电子学自动测试 10 个 组件 ${ }^{[1]}$ 。在此基础之上, 着手研发第二代通用组件的 同时改进第一代。

\section{SADA 组件的应用}

20 世纪 90 年代, 美国国防部为了标准化美军使 用的二代前视红外 (Forward Looking Infrared, FLIR) 系统，在一代通用组件的基础上制定了标准的先进杜 瓦组件 (Standard Advanced Dewar Assembly, SADA) 系列, 由红外焦平面阵列、杜瓦、指令/控制电子单元 和低温制冷机组成, 分为 SADA I、SADA II 和 SADA III 三类 ${ }^{[5]}$ 。SADA I 用于美国 Apache 直升机光电火控 系统等高性能热成像系统, SADA II 用于 M2A3 Bradley 步兵战车、M1A2 Abrams 主战坦克、美国陆 军远距离目标获取侦察监视系统 (Long Range Advanced Scout Surveillance System, LRAS3）等中性 能热成像系统, SADA III 用于标枪指挥发射装置、单 兵肩扛式标枪导弹等紧凑型或低性能热成像系统。

2005 年 12 月, Raytheon 公司与 DRS 公司签订了 价值 180 万美元的合同, 用于生产安装在 M2A3 Bradley 步兵战车和 M1A2 Abrams 主战坦克上的 SADA II 组件。2008 年 2 月, Raytheon 和 DRS 公司 又签订了另外两份合同，总价值 4880 万美元，用于 生产安装在 M1A2 主战坦克、M2A3 步兵战车和 LRAS3 系统中的 SADA II 组件 ${ }^{[6]}$ 。

SADA II 使用了 $480 \times 4 \mathrm{MCT}$ 长波红外探测器。 用于 Javelin 指挥发射装置平台的 SADA IIIA 和 SADA IIIB 分别使用了 $240 \times 2 \mathrm{MCT}$ 和 $288 \times 1 / 240 \times 4 \mathrm{MCT}$ 焦平面, SADA IIIB 的探测器张角为 $44^{\circ} \times 51^{\circ}$ (水平 角 $\times$ 俯仰角)。为了增加灵敏度和作用距离, SADA II 在时间延迟积分内用了 6 个光伏探测器。SADA IIIB 在时间延迟积分内用了 4 个光伏探测器。

SADA IIIA 和 SADA IIIB 分别被封装在一个带有 线性驱动斯特林循环制冷机和指令/控制电子元件的 集成式杜瓦组件中。单片长波红外器件由探测器芯片 和硅读出电路芯片集成在一起, 包含盲元剔除和自动 增益补偿功能。表 1 为 SADA II 和 SADA III 系列通 用组件的技术参数 ${ }^{[6]}$ 。

目前, Apache 直升机 “箭头” 光电火控系统集成 了基于 SADA I 的 M-TADS (Modernized Target Acquisition Designation Sight)/M-PNVS（Modernized Pilot Night Vision Sensor）热成像接收器。Bradley 步 兵战车安装了基于 SADA II 的增强型 Bradley 目标获 取子系统 (Improved Bradley Acquisition Subsystem, IBAS）和 IBAS BLOCK2 子系统, IBAS BLOCK2 基 于 IBAS 产生, 主要改进在于能够高清、高分辨率彩 色成像, 优于过去的黑白成像。安装在美军密集阵火 炮系统上的舰载热像仪 (Phalanx Thermal Imager, PTI）也使用了 SADA II。表 2 是 DRS 公司推出的基 于 SADA 系列通用组件的 TADS/PNVS 接收器、IBAS 子系统、IBAS BLOCK2 子系统和 PTI 热像仪的技术 参数 ${ }^{[7-10]}$ 。 
表 1 SADA II 和 SADA III 系列通用组件的技术参数

Table 1 Specifications of SADA II and SADA III-series

\begin{tabular}{|l|l|l|l|}
\hline & SADA II & SADA IIIA & \multicolumn{2}{|l|}{ SADA IIIB } \\
\hline Detector & MCT photovoltaic & \multicolumn{2}{|l|}{} \\
\hline Spectral range & $8-12 \mu \mathrm{m}$ & $240 \times 2$ & $288 \times 1$ or $240 \times 4$ \\
\hline Array format & $480 \times 4$ & & \\
\hline Min resolvable temperature & $0.02^{\circ} \mathrm{C}$ & & \\
\hline Scene dynamic range & $-33^{\circ} \mathrm{C}-+87^{\circ} \mathrm{C}$ & & $20-83 \mathrm{kHz}$ \\
\hline Sample rate & $20-160 \mathrm{kHz}$ & & $0.5-5.0 \mathrm{MHz}$ \\
\hline Video output rate & $0.5-5.0 \mathrm{MHz}$ & & $\mathrm{f} / 2.31 \mathrm{standard}$ \\
\hline Coldshield f-number & $\mathrm{f} / 2.5 \mathrm{standard}$ & & $12 \mathrm{~W}$ nominal, $24 \mathrm{~W}$ max \\
\hline Cooler input power & $20 \mathrm{~W}$ nominal, $60 \mathrm{~W}$ max & & $1.5 \mathrm{~W}$ \\
\hline Detector electronics power & $4 \mathrm{~W}$ & & $11.532 \mathrm{~cm} \times 6.045 \mathrm{~cm}$ \\
\hline $\begin{array}{l}\text { Dimensions } \\
\text { (Dewar assembly) }\end{array}$ & $8.687 \mathrm{~cm} \times 10.617 \mathrm{~cm} \times$ & & $0.295 \mathrm{~kg}$ \\
\hline Dewar assembly weight & $10.668 \mathrm{~cm}$ & $0.635 \mathrm{~kg}$ & \\
\hline Cooler weight & $1.905 \mathrm{~kg}$ & & $0.454 \mathrm{~kg}$ \\
\hline
\end{tabular}

表 2 DRS 公司推出的基于 SADA 系列组件的 TADS/PNVS 接收器、IBAS 子系统、IBAS BLOCK2 子系统和 PTI 热像仪的技术 参数

Table 2 Specifications of SADA-series based TADS/PNVS receiver, IBAS, IBAS BLOCK2 and PTI developed by the DRS company

\begin{tabular}{|c|c|c|c|c|}
\hline & TADS/PNVS & IBAS & IBAS BLOCK2 & PTI \\
\hline Detector & SADA I & SADA II & SADA II & SADA II \\
\hline Array format & $480 \times 5$ & $480 \times 4, \mathrm{f} / 2.50$ & $480 \times 4, f / 250$ & $640 \times 480$ \\
\hline Spectral response & $8-12 \mu \mathrm{m}$ & & & \\
\hline \multirow{2}{*}{ FOV } & M-TADS: $20.0^{\circ} \times 26.7^{\circ}$ & $\begin{array}{l}\text { WFOV: } 13.33^{\circ} \pm 0.67^{\circ} \mathrm{H} \\
7.5^{\circ} \pm 0.38^{\circ} \mathrm{V}\end{array}$ & $\begin{array}{l}\text { WFOV: } 13.33^{\circ} \pm 0.67^{\circ} \mathrm{H} \\
7.5^{\circ} \pm 0.38^{\circ} \mathrm{V}\end{array}$ & $\begin{array}{l}\text { WFOV: } 6.0^{\circ} \mathrm{H} \\
3.9^{\circ} \mathrm{V}\end{array}$ \\
\hline & M-PNVS: $23.0^{\circ} \times 41.9^{\circ}$ & $\begin{array}{l}\text { NFOV: } 3.56^{\circ} \pm 0.18^{\circ} \mathrm{H} \\
2.0^{\circ} \pm 0.10^{\circ} \mathrm{V}\end{array}$ & $\begin{array}{l}\text { NFOV: } 3.56^{\circ} \pm 0.18^{\circ} \mathrm{H} \\
2.0^{\circ} \pm 0.10^{\circ} \mathrm{V}\end{array}$ & $\begin{array}{l}\text { NFOV: } 2.0^{\circ} \mathrm{H} \\
1.3^{\circ} \mathrm{V}\end{array}$ \\
\hline \multicolumn{5}{|l|}{ General } \\
\hline MTBF & 3912 hours & 330 hours & & 2000 hours \\
\hline Service life & $>10,000$ hours over 20 years & & & \\
\hline \multicolumn{5}{|l|}{ Mechanical } \\
\hline Weight & $\begin{array}{l}\text { M-TADS: }<7.7 \mathrm{~kg} \\
\text { M-PNVS: }<8.85 \mathrm{~kg}\end{array}$ & & & $<28.58 \mathrm{~kg}$ \\
\hline \multirow{2}{*}{ Power } & $\begin{array}{l}\text { Cooler: +28 V, } 336 \mathrm{~W} \text { (Peak) } \\
95 \mathrm{~W} \text { (Steady state) }\end{array}$ & & & \multirow{2}{*}{ 28VDC, $90 \mathrm{~W}$} \\
\hline & $\begin{array}{l}\text { Receiver: +/-135 V, } 75 \text { W (Peak) } \\
40 \text { W (Steady state) }\end{array}$ & & & \\
\hline
\end{tabular}

\section{3 二代前视红外水平技术集成}

1993 年 2 月, 为满足将热像仪批量集成到现有和 未来作战/非作战车辆的光电瞄准镜上的需求, 美国陆 军提出二代 FLIR 的水平技术集成计划, 即二代 FLIR 通用组件计划, 专用于研发和集成二代 FLIR 通用组 件, 探测器元件为 SADA II ${ }^{[6]}$ 。通过对比提供相似性能 的多种专用系统和应用于多种平台的单一系统的制造 成本, HTI FLIR 实现了制造研发阶段的成本节约 ${ }^{[11]}$ 。 为了与坦克炮或导弹系统的有效作用距离兼容, HTI 在一代通用组件的基础上增加了 1.5 倍以上的探测距 离和 2 倍的识别距离。该项目的目标是开发一款名为 HTI NV-80 套件 B (HTI NV-80 B-Kit, 以下简称为套 件 B) 的标准热像仪, 通过利用名为套件 A（A-Kits） 的车辆专用集成部件来实现在 M1A2 Abrams 主战坦 克、M2A3 Bradley 步兵战车和 LRAS3 系统中的安装。
安装在 M1A2 Abrams 主战坦克和 XM8 轻型坦克上的 套件 B 需要对现有车型进行改造。安装在 M2A3 Bradley 步兵战车和 LRAS3 系统中的套件 B 是全新开 发的二代 HTI FLIR 系统。

套件 B 可在 $30 \mathrm{~Hz}$ 非隔行扫描和 $60 \mathrm{~Hz}$ 电子交错 扫描两种模式下工作。纵横比为 16:9, 宽视场为 $7.5^{\circ}$ $\times 13.3^{\circ}$, 窄视场为 $2.0^{\circ} \times 3.56^{\circ}$, 具有 2 倍和 4 倍的电 子变焦能力, 提供两种数字输出和两种模拟输出。与 一代通用组件相比, 系统性能可将识别距离增加 2 倍、 确认距离增加约 50\%。

套件 B 分为两个外场可更换单元: 传感器组件和 通用电子单元。传感器组件由远焦系统组件、热像仪 组件和探测器/制冷机组件构成。光机组件的第一部分 是远焦系统组件, 第二部分是热像仪组件, 其中探测 器/制冷机组件使用了 SADA II 探测器和线性驱动制 冷机。通用电子单元包含视频处理器电路板、视频转 
换器电路板、接口控制电路板、电源、备用电源、帧 积分/对比度增强电路板和未来应用的扩展插槽。

美军项目管理对低速初期生产 (Low Rate Initial Production, LRIP) 的定义是指系统 (不包括舰船和 卫星) 以有限数量进行的生产, 其目的是为使用试验 和评价提供代表批生产的试件, 建立初步生产基地, 使生产率有序增长、以便使用试验成功完成后进入大 批量生产。文献[11]介绍的 HTI 项目包括两个 LRIP 合同, 一个是套件 B 的 LRIP 合同, 另一个是 M1A2 主战坦克瞄准镜的 LRIP 合同。具体数量为: 9 套用 于 M1A2 主战坦克的车长独立热像仪; 11 套用于 M1A2 主战坦克的炮长主瞄准镜的热成像系统; 12 套 用于 M2A3 车长独立观察装置的套件 B; 6 套用于 M2A3 增强型 Bradley 目标获取系统的套件 B; 9 套用 于 M8 装甲火炮系统的炮长主瞄准子系统的热成像系 统; 4 套用于 LRAS3 的带有远距离光学系统的套件 B; 2 套用于合格性测试的套件 B。

1994 年 7 月, HTI 项目获得“里程碑 I/II (Milestone I/II）”许可。第一批套件 B 从 1994 年 11 月开始交 付美国军方, 1997 年 3 月底, 共生产完成 61 套套件 B/瞄准镜。经过 20 多年的发展, HTI 技术已十分成熟, 覆盖范围包括制冷型和非制冷型探测器, 车辆平台包 括作战和非作战车辆。例如, DRS 公司基于 HTI 技术 开发的驾驶员视觉增强器 (DVE) 的月产能达到 2500 套, 可在 30 天内装备美军的一个旅级单位 ${ }^{[12]}$ 。

据公开资料报道, 美国陆军与 DRS 公司签订了 一项总额为 6700 万美元的合同, 指定后者为美军 M1A1 Abrams 主战坦克、Bradley 步兵战车、Stryker 轮式装甲车提供基于 HTI 二代 FLIR 技术的套件 $1 \mathrm{~A} /$ 套件 1B（SG-FLIR Block 1 A/B-kits）。该合同将于 2026 年完成 ${ }^{[13]}$ 。

\section{4 通用组件技术中的制冷机}

为满足二代 FLIR 系统不同的制冷需求, 美国夜 视和电子传感器部门 (NVESD) 研发了一系列线性驱 动制冷机。与具有相似性能的制冷机相比, 成本较低 (10000 美元以下)、寿命较短 (平均故障时间为 4000 $8000 \mathrm{~h}$ ) 、冷却时间较快（在不到 $15 \mathrm{~min}$ 的时间内温 度达到 $80 \mathrm{~K}$ ）, 会受到温度过高或过低、较大程度的 机械冲击和工作环境中常见的振动等影响 ${ }^{[5,14-15]}$ 。为 了改进旋转式制冷机可靠性低、多轴振动、噪声过大、 缺少温度稳定性等缺点, 线性驱动制冷机提高了可靠 性、降低了成本、改善了平均故障间隔时间。

美国国防部定义的线性驱动制冷机系列的功率 有 $0.15 \mathrm{~W} 、 1.0 \mathrm{~W} 、 1.5 \mathrm{~W}$ 和 $1.75 \mathrm{~W}$, 是斯特林循环、
双对置活塞、线性驱动单元, 与 SADA 系列的集成通 过用于驱动线性电机和冷端温度控制的外部或者内 部控制电子单元来实现。

$0.15 \mathrm{~W}$ 线性驱动制冷机用于便携式反坦克导弹 系统的 Javelin 指挥发射单元。因为便携式系统由电池 供电, 制冷机效率较为重要, 所以 $0.15 \mathrm{~W}$ 制冷机的设 计使膨胀器靠近压缩机, 可通过非常短的传输线实 现。1.0 W 线性驱动制冷机应用于美国陆军二代 FLIR 水平技术集成项目。因为应用范围较广, 所以 $1.0 \mathrm{~W}$ 制冷机主要专注于限定制造商、降低成本和提升可靠 性。1.75 W 制冷机最初用于高性能 FLIR 系统, 例如, Apache 直升机、现已取消的 Comanche 直升机和量子 阱热成像系统。由于重量原因, 已被重量更轻的 $1.5 \mathrm{~W}$ 制冷机所替代。1.5 W 制冷机用于高性能 FLIR 系统, 在 $1.0 \mathrm{~W}$ 制冷机的基础上改进而来, 其冷却或者制冷 量的需求都超过了 $1.0 \mathrm{~W}$ 制冷机。重量较轻, 是 Apache 直升机使用的 $1.75 \mathrm{~W}$ 制冷机的替代件。

\section{5 结束语}

在军用红外技术应用的早期阶段, 由于需要装备 热成像系统的武器平台种类繁多, 各有差异, 如果每 个武器平台研发一套热成像系统, 将形成数量众多、 功能大同小异的系统组件, 导致研发成本上升, 产品 缺乏通用性和互换性。通用组件符合标准化、规范化 和通用化趋势, 预留进一步发展的空间。美军红外项 目一般以成熟的通用组件技术为基础实现工程化。通 用组件虽然减少了工程上选择不同方案的余地，但提 高了可生产性，降低了工程制造开发的成本。

通用组件的初衰是为了控制成本, 它是以牺牲组 件的灵活性、先进性、独特性为代价的。通用组件技 术实施以后, 红外技术发展迅速, 一方面探测器的规 格从通用组件时的几个线列 (例如, $288 \times 4 、 480 \times 4$ 等) 逐步发展到半帧、全帧，甚至百万像元的高清格 式焦平面阵列。另一方面, 随着微电子技术的发展, 实现同样功能所需的硬件体积变小、重量变轻, 使得 原来需要用几块电路板实现的功能可在一块电路板 上实现，在控制成本的同时可以实现组件的灵活定 制, 这种趋势导致对通用性的需求逐渐弱化，反映在 英文文献中就是 Common Module 的提法逐渐被 Module 取代, 但是这并不意味着通用化的思想被扬 弃, Common 依然深刻地渗透在 Module 的设计研发 过程中, 只是从大 Common 变为小 Common。

\section{参考文献:}

[1] 张志福. 热成象技术发展剖析[J]. 红外技术, 1987, 9(2): 1-22. 
ZHANG Zhifu. Development and Analysis of Thermal Imaging Technology[J]. Infrared Technology, 1987, 9(2): 1-22.

[2] 张坤杰. 美国军用红外通用组件技术的发展 [J]. 云光技术, 2019, 51(1): 6-13

ZHANG Kunjie. Military Development of Infrared Common Module in the United States[J]. Yunguang Jishu, 2019, 51(1): 6-13.

[3] Glenn M Cuthbertson. TICM II Stretch the UK Thermal Imaging Common Module Class II Enhancement Programme[C]/SPIE: Optical Systems for Space and Defense, 1989, 1191: 509-521.

[4] Glenn M Cuthbertson. Stretch TICM - The UK Thermal Imaging Common Module Class II Enhancement Programme[C]//SPIE: Infrared Technology XV, 1989, 1157: 185-199.

[5] Garin S. Tate. Linear-Drive Cryocoolers for the Department of Defense Standard Advanced Dewar Assembly[C]//SPIE, 2005, 5783: 138-144.

[6] Raytheon Network Centric Systems, DRS Technologies Inc., Reconnaissance, Surveillance and Target Acquisition (RSTA) group. Standard Advanced Dewar Assembly (SADA)[M]. Jane's Electro-Optic Systems, 2013-2014.

[7] Leonardo DRS Company. ARROWHEAD[M/OL]. [2018-8-13]. https:// www.leonardodrs.com/media/3298/arrowhead_datasheet.pdf

[8] Leonardo DRS Company. IBAS[M/OL]. [2018-9-19]. https://www. leonardodrs.com/media/3295/ibas_datasheet.pdf

[9] Leonardo DRS Company. IBAS BLOCK2[M/OL]. [2018-10-19]. https:// www.leonardodrs.com/media/4476/ibas_block2_datasheet.pdf

[10] Leonardo DRS Company. Phalanx Thermal Imager[M/OL]. [2018-11-20]. https://www.leonardodrs.com/products-and-services/ phalanx-thermal-imager-pti/

[11] LTC.(P) Joseph P. Mackin, Douglas K. Wiltsie. The U.S. Army Development of the Second Generation Horizontal Technology Integration Forward Looking Infrared (FLIR)[C]//SPIE, 1996, 2764: 188-194.

[12] Raytheon Missile Systems (formerly Raytheon Network Centric Systems), DRS Technologies Inc, Reconnaissance, Surveillance and Target Acquisition (RSTA) segment. AN/VAS-5 Driver's Vision Enhancer (DVE) [M]. Jane's Electro-Optic Systems, 2013-2014.

[13] Army Technology. Leonardo DRS to deliver SG-FLIR kits for US Army combat vehicles [EB/OL]. [2019-4-1]. https://www.army-technology. com/news/leonardo-sg-flir-kits-us-vehicles/.

[14] Jeroen Mullié, Willem vd Groep, Peter Bruins, et al. Improvement of Cooldown time of LSF9599 Flexure Bearing SADA cooler[C]//SPIE, 2006, 6206: 620632-1-620623-7.

[15] 许红. 美国战术用线性斯特林制冷机进展[J]. 红外技术, 2009, 31(7): 420-423.

XU Hong. Development of the Tactical Linear Stirling Cryocooler in USA[J]. Infrared Technology, 2009, 31(7): 420-423.

\section{( 上接第 682 页)}

[12] 齐海生, 荣传振, 肖力铭, 等. 基于双树复小波变换与引导滤波的红 外与可见光图像融合算法 [J]. 通信技术, 2019, 52(2): 78-84.

QI Haisheng, RONG Chuanzhen, XIAO Liming, et al. Development and performance evaluation of infrared and visual image fusion technology[J]. Communication Technology, 2019, 52(2): 78-84.

[13] LIANG Z T, XU J, ZHANG D, et al. A Hybrid 11-10 Layer Decomposition Model for Tone Mapping[C]//IEEE Conference on Computer Vision and Pattern Recognition, 2018, 7: 1-9.

[14] 周强, 赵巨峰, 冯华君, 等. 非下采样剪切波的红外偏振图像融合[J]. 浙江大学学报: 工学版, 2014, 48(8): 1508-1516.

ZHOU Qiang, ZHAO Jufeng, FENG Huajun, et al. Infrared polarization image fusion with non-sampling Shearlets[J]. Journal of Zhejiang University: Engineering Science, 2014, 48(8): 1508-1516.

[15] ZHANG Lei, YANG Fengbao, JI Linna, et al. Infrared polarization and intensity image fusion algorithm based on transfer of image feature[J]. Automatic Control and Computer Sciences, 2018, 52(2): 135-145.

[16] 安富, 杨风暴, 济素珍, 等. 基于局部能量与模糊逻辑的红外偏振图
像融合[J]. 红外技术, 2012, 34(10): 573-583.

AN Fu, YANG Fengbao, LIN Suzhen, et al. Infrared Polarization Images Fusion Based on Local Energy and Fuzzy Logic[J]. Infrared Technology, 2012, 34(10): 573-583.

[17] Ratliff B M, Tyo J S, Boger J K, et al. Dead pixel replacement in LWIR microgrid polarimeters[J]. Optics Express, 2007, 15(12): 7596-7609.

[18] LIN S Z, WANG D J, ZHU X H, et al. Fusion of infrared intensity and polarization images using embedded multi-scale transform[J]. Optik International Journal for Light and Electron Optics, 2015, 126(24): 5127-5133.

[19] LI S , KANG X , HU J . Image Fusion With Guided Filtering[J]. IEEE Transactions on Image Processing, 2013, 22(7): 2864-2875.

[20] Aslantas V, Bendes E. A new image quality metric for image fusion: The sum of the correlations of differences[J]. International Journal of Electronics and Communications, 2015, 69(3): 1890-1896. 